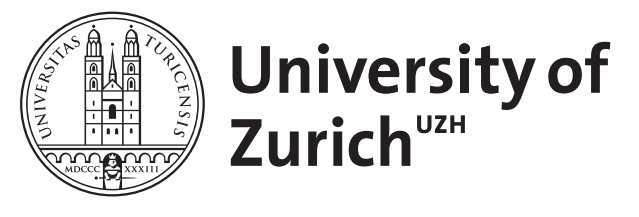

\title{
On the Reynolds lubrication equation
}

\author{
Chipot, M
}

\begin{abstract}
Let $\Omega$ be a bounded open set in R 2 with "smooth" boundary $\Omega . \Omega$ is the region where two solid bodies are in contact. These two solids are moving and the average of their velocities is denoted by $\mathrm{V}=(\mathrm{V} 1, \mathrm{~V} 2)$. The pressure $\mathrm{p}=\mathrm{p}(\mathrm{X})$ which develops in a fluid layer confined between these two bodies satisfies the so- called Reynolds lubrication equation: (1) $\cdot(\mathrm{h} 3 \mathrm{p})=6 \mathrm{~V} \cdot(\mathrm{h}) \mathrm{in} \Omega(2) \mathrm{p}=\mathrm{p}$ a on $\Omega \cdot$ Here is the density of the fluid, $>0$ its dynamic viscosity, $h=h(X)$ is the distance between the two bodies and $\mathrm{p}$ a 0 is the given ambient pressure. We will assume all along that $\mathrm{h}$ is a Lipschitz continuous function such that $(3) 0<\mathrm{h} 1 \mathrm{~h}(\mathrm{X}) \mathrm{h} 2 \mathrm{a} \cdot \mathrm{e} \cdot \mathrm{X}=(\mathrm{x}, \mathrm{y}) \Omega(4)|\mathrm{h}(\mathrm{X})| \mathrm{Ha} \cdot \mathrm{e} \cdot \mathrm{X}=(\mathrm{x}, \mathrm{y}) \Omega$ where h 1 , h 2 , $\mathrm{H}$ are positive constants. When the fluid is incompressible, i.e. is a positive constant, existence and uniqueness for a solution to (1), (2) is a trivial matter since the equation is linear. In the compressible case when $(5)=(\mathrm{p})$ the problem becomes more challenging. The paper is organized as follows: in Section 1 we investigate the problem of existence of a solution for (1), (2) when is given by (5). In Section 2 we consider a case where (1), (2) reduces to a one dimensional problem and we study the question of uniqueness as well as the shape of the solution. Finally, in a third section we give some extensions of the results of Section 2 in higher dimension.
\end{abstract}

DOI: https://doi.org/10.1016/0362-546X(88)90023-5

Posted at the Zurich Open Repository and Archive, University of Zurich ZORA URL: https://doi.org/10.5167/uzh-22949

Journal Article

Originally published at:

Chipot, M (1988). On the Reynolds lubrication equation. Nonlinear Analysis: Theory, Methods Applications, 12(7):699-718.

DOI: https://doi.org/10.1016/0362-546X(88)90023-5 


\section{ON THE REYNOLDS LUBRICATION EQUATION \\ BY \\ M. CHIPOT}

IMA Preprint Series 非 206

January 1986

\section{INSTITUTE FOR MATHEMATICS AND ITS APPLICATIONS UNIVERSITY OF MINNESOTA \\ 514 Vincent Hall 206 Church Street S.E. Minneapolis, Minnesota 55455}


On the Reynolds Lubrication Equation

\author{
M. Chipot \\ Institute for Mathematics and its Applications \\ 514 Vincent $\mathrm{Hall}$ \\ 206 Church St. S.E. \\ University of Minnesota \\ Minneapolis, MN 55455 (U.S.A.) \\ and \\ Université de Metz \\ Département de Mathématiques \\ Ile du Saulcy, 57045 Metz - Cedex (FRANCE)
}




\section{Introduction}

Let $\Omega$ be a bounded open set in $\mathbb{R}^{2}$ with "smooth" boundary $\partial \Omega . \Omega$ is the region where two solid bodies are in contact. These two solids are moving and the average of their velocities is denoted by $\underline{V}=\left(V_{1}, V_{2}\right)$. The pressure $p=p(X)$ which develops in a fluid layer confined between these two bodies satisfies the so-called Reynolds lubrication equation:

$$
\begin{array}{rlrl}
\nabla \cdot\left(h^{3} \rho \nabla p\right) & =6 \underline{\underline{V}} \cdot \nabla(\rho h) \quad \text { in } \Omega \\
p & =P_{a} \text { on } \partial \Omega . &
\end{array}
$$

Here $\rho$ is the density of the fluid, $\mu>0$ its dynamic viscosity, $h=h(X)$ is the distance between the two bodies and $P_{a} \geqslant 0$ is the given ambient pressure. (see $[1][7][8])$.

We will assume all along that $h$ is a Lipschtz continuous function such that

$$
\begin{aligned}
0<h_{1} \leqslant h(x) \leqslant h_{2} & \text { a.e. } & x=(x, y) \in \Omega \\
|\nabla h(x)| \leqslant H & \text { a.e. } & x=(x, y) \in \Omega
\end{aligned}
$$

where $h_{1}, h_{2}, H$ are positive constants.

When the fluid is incompressible, - i.e. when $\rho$ is a positive constant, existence and uniqueness for a solution to (1) (2) is a trivial matter since the equation is linear. In the compressible case when

$$
\rho=\rho(p)
$$

the problem becomes more challenging. In [3] (see also [4], [5]) the problem has been investigated in the case where

$$
\rho(p)=p
$$

that is to say when the lubricating fluid obeys the isothermal perfect gas relation (after rescaling). In this paper we are going to examine the general case. However, most of our results are new even in the case where (6) holds. 
The paper is organized as follows: in section 1 we investigate the problem of existence of a solution for (1)(2) when $\rho$ is given by (5). In section 2 we consider a case where (1),(2) reduces to a one dimensional problem and we study the question of uniqueness as well as the shape of the solution. Finally, in a third section we give some extensions of the results of section 2 in higher dimension.

\section{Existence}

Assume that we are looking for a positive solution $p$ of

$$
\begin{gathered}
\nabla \cdot\left(h^{3} \rho(p) \nabla p\right)=\sigma_{\mu} \underline{V} \cdot \nabla(\rho(p) h) \text { in } \Omega . \\
p=p_{a} \geqslant 0 \text { on } \partial \Omega .
\end{gathered}
$$

For physical reasons it is natural to assume that $\rho$ satisfies:

$\rho$ is continuous, non decreasing and is such that $\rho(0)=0, \rho(s)>0 \quad \forall s>0$.

Clearly, under this assumption, the function $R$ defined by

$$
R(s)=\int_{0}^{s} \rho(t) d t
$$

is one-to-one from $[0,+\infty)$ onto itself. Let us denote its inverse by $R^{-1}$. If $p>0$ is a solution of $(7)(8)$ (for instance in the classical sense when the data are smooth) then

$$
u=R(p)
$$

is a solution of

$$
\begin{gathered}
\nabla \cdot\left(h^{3} \nabla u\right)=\underline{v} \cdot \nabla\left(6 \mu \cdot h \cdot \rho \circ R^{-1}(u)\right) \text { in } \Omega \\
u=\phi \text { on } \partial \Omega
\end{gathered}
$$

where we have set $\phi=R\left(P_{a}\right)$. Conversely, if $u>0$ is a solution of (12)(13) then $p=R^{-1}(u)$ is a solution of $(7)(8)$. So setting 


$$
\beta(s)=6_{\mu} \cdot \rho \circ R^{-1}(s)
$$

we are going to consider the problem

$$
\begin{aligned}
\nabla \cdot\left(h^{3} \nabla u\right) & =\underline{v} \cdot \nabla(h \beta(u)) \quad \text { in } \Omega . \\
u & =\phi \text { on } \partial \Omega .
\end{aligned}
$$

(If we do not know a priori that $u$ is non negative we can extend $\beta$ by 0 when $S$ is negative) The assumptions on $\beta$ will be:

$$
\beta \text { is a continuous function }
$$

$$
\exists C>0 \text { such that }|\beta(s)| \leqslant \frac{\sqrt{v h_{1}^{3}}}{2 h_{2}|\underline{v}|}|s|+c \quad \forall s \in \mathbb{R}
$$

where $h_{1}, h_{2}$ are the constants defined in (3), $|\underline{V}|$ denotes the Euclidean norm of $\underline{v}$ and $\nu$ is the smallest eigenvalue of the problem

$$
\begin{aligned}
-\Delta W & =\nu W \text { in } \Omega \\
W & =0 \text { on } \partial \Omega .
\end{aligned}
$$

Remark 1: It is very easy to check that the above assumptions hold for instance for $s \geqslant 0$ when $\rho$ is given by

$$
\rho(s)=C s^{\alpha}
$$

where $C, \alpha$ are two positive constants. More precisely in this case we have

$$
\beta(s)=6_{\mu} C\left(\frac{\alpha+1}{C}\right)^{\frac{\alpha}{\alpha+1}} \frac{\alpha}{\alpha+1} .
$$

Let us denote by $H^{1}(\Omega), H_{0}^{1}(\Omega)$ the usual Sobolev spaces on $L^{2}(\Omega)$ (see for instance [6]). For $\phi \in H^{1}(\Omega)$ we will say that $u$ is a weak solution of (15)(16) if and only if:

$$
\begin{gathered}
u-\phi \in H_{0}^{1}(\Omega) \\
\int_{\Omega}\left(h^{3} \nabla u \cdot \nabla \xi-h \beta(u) \underline{v} \cdot \nabla \xi\right) d x=0 \quad \forall \xi \in H_{0}^{1}(\Omega) .
\end{gathered}
$$


Remark 2: Note that when $u \in H^{1}(\Omega)$ we have $\beta(u) \in L^{2}(\Omega)$ and (23) makes sense $($ see $(3)(17)(18))$

Then we have:

Theorem 1: Let $\phi \in H^{1}(\Omega)$ and assume that $(3)(17)(18)$ hold. Then there exists a weak solution of $(15)(16)$

Proof: For $v \in L^{2}(\Omega)$ let us denote by $u=T(v)$ the solution of the Dirichlet problem:

$$
\begin{gathered}
u-\phi \in H_{0}^{1}(\Omega) \\
\int_{\Omega}\left(h^{3} \nabla u \cdot \nabla \xi-h \beta(v) \underline{v} \cdot \nabla \xi\right) d x=0 \quad v \xi \in H_{0}^{1}(\Omega) .
\end{gathered}
$$

Moreover set

$$
B_{R}=\left\{\left.v \in L^{2}(\Omega)|| v\right|_{2} \leqslant R\right\}
$$

where $\mid l_{2}$ denotes the usual norm on $L^{2}(\Omega)$.

First, we claim that for $R$ large enough, $T$ maps $B_{R}$ into $B_{R}$. Indeed if we substitute $\xi=u-\phi$ into (25) we obtain:

$$
\int_{\Omega} h^{3}|\nabla(u-\phi)|^{2} d x=\int_{\Omega}\left[h \beta(v) \underline{v} \cdot \nabla(u-\phi)-h^{3} \nabla \phi \cdot \nabla(u-\phi)\right] d x .
$$

( $\mid$ denotes the usual Euclidean norm). Using (3) and (18) we get for some constant $C$

$$
h_{1}^{3} \int_{\Omega}|\nabla(u-\phi)|^{2} d x \leqslant \sqrt{v} h_{1}^{3} \int_{\Omega}\left(\frac{1}{2}|v|+C(1+|\nabla \phi|)\right)|\nabla(u-\phi)| d x \quad .
$$

Hence by Cauchy-Scharz inequality:

$$
\int_{\Omega}|\nabla(u-\phi)|^{2} \mathrm{dx} \leqslant v \int_{\Omega}\left(\frac{1}{2}|v|+c+c|\nabla \phi|\right)^{2} \mathrm{dx}
$$




$$
\int_{\Omega}|u-\phi|^{2} d x \leqslant v^{-1} \int_{\Omega}|\nabla(u-\phi)|^{2} d x .
$$

leads to:

$$
\int_{\Omega}|u|^{2} \mathrm{dx} \leqslant \frac{1}{2} \int_{\Omega}|v|^{2} \mathrm{dx}+\mathrm{C}
$$

for some constant $C$. If now we choose $R^{2} \geqslant 2 C$ it is clear that for $v \in B_{R}$ we have by (27)

$$
|u|_{2}^{2} \leqslant \frac{1}{2} R^{2}+C \leqslant R^{2}
$$

and thus $T$ maps $B_{R}$ into $B_{R}$. From (25) and the fact that $H^{1}(\Omega)$ is compactly embedded in $L^{2}(\Omega)$, we see that $T\left(B_{R}\right)$ is precompact in $B_{R}$. If we assume that $T$ is continuous, then, by Schauder fixed point theorem, $T$ has a fixed point in $B_{R}$ which is a weak solution of (15), (16). So it remains only to prove that $T$ is continuous. Let $v_{n}$ be a sequence in $B_{R}$ such that $v_{n} \rightarrow v$ in $L^{2}(\Omega)$. We have to show that $T\left(v_{n}\right) \rightarrow T(v)$ in $L^{2}(\Omega)$. For this, thanks to (26) and the fact that $H^{1}(\Omega)$ is compactly embedded in $L^{2}(\Omega)$, it is enough to show that $T(v)$ is the only limit point of the sequence $T\left(v_{n}\right)$. So let us assume that a subsequence $T\left(v_{n_{k}}\right) \rightarrow u$ when $k \rightarrow+\infty$. We can extract a subsequence, still denoted by $n_{k}$, such that

$$
\begin{aligned}
& v_{n_{k}} \rightarrow v \text { a.e. in } \Omega \\
& u_{n_{k}}=T\left(v_{n_{k}}\right) \rightarrow u \text { in } H^{1}(\Omega) .
\end{aligned}
$$

The set $\left\{u \in H^{1}(\Omega) \mid u-\phi \in H_{0}^{l}(\Omega)\right\}$ is closed and convex and so is weakly closed in $H^{1}(\Omega)$. Thus $u_{n_{k}}-\phi \in H_{0}^{l}(\Omega)$ implies that $u-\phi \in H_{0}^{1}(\Omega)$. Now passing to the limit in (25) i.e. in

$$
\int_{\Omega}\left(h^{3} \nabla u_{n_{k}} \nabla \xi-h \beta\left(v_{n_{k}}\right) \underline{v} \cdot \nabla \xi\right) d x=0 \quad \forall \xi \in H_{0}^{1}(\Omega)
$$


we get

$$
\int_{\Omega}\left(h^{3} \nabla u \cdot \nabla \xi-h \beta(v) \underline{\underline{v}} \cdot \nabla \xi\right) d x=0 \quad \forall \xi \in H_{0}^{1}(\Omega)
$$

Thus $u=T(v)$ and the proof is complete.

(To show that

$$
\int_{\Omega} h \beta\left(v_{n_{k}}\right) \underline{V} \cdot \nabla \xi d x \rightarrow \int_{\Omega} h \beta(v) \underline{V} \cdot \nabla \xi d x
$$

we have to use Vitali's theorem. Indeed

$$
h \beta\left(v_{n_{k}}\right) \underline{V} \cdot \nabla \xi \rightarrow h \beta(v) \underline{V} \cdot \nabla \xi \quad \text { a.e. in } \Omega \text {. }
$$

Moreover for any measurable set $E$ we have by (18) and for some constant C

$$
\begin{aligned}
\int_{E}\left|h \beta\left(v_{n_{k}}\right) \underline{v} \cdot \nabla \xi\right| d x & \leqslant C \int_{E}\left|v_{n_{k}}\right||\nabla \xi| d x+C \int_{E}|\nabla \xi| d x \\
& \leqslant C \int_{E}\left|v_{n_{k}}-v\right||\nabla \xi|+C \int_{E}(|v|+1)|\nabla \xi| d x \\
& \leqslant C|| \nabla \xi||_{2}\left|v_{n_{k}}-v\right|_{2}+C \int_{E}(|v|+1)|\nabla \xi| d x .
\end{aligned}
$$

Since $v \in L^{2}(\Omega)$ and $\left|v_{n_{k}}-v\right|_{2} \rightarrow 0$ with $k \rightarrow+\infty$ this inequality proves the uniform integrability of the sequence $h \beta\left(v_{n_{k}}\right) \underline{v} \cdot \nabla \xi$ and (28) follows. see [9] p. 143)

\section{The One Dimensional Case}

When the dimension of $\Omega$ in the $x$ direction is small compared to its dimension in $y$, when $h$ doesn't depend on $y$ and $\underline{V}=\left(v_{1}, 0\right)$ then finding the pressure $p$ which develop between the two bodies can be reduced to solving a one dimensional problem. More precisely for some $\Omega=(a, b)$ this leads to finding $p=p(x) \geqslant 0$ such that

$$
\begin{gathered}
\left(h^{3} \rho(p) p^{\prime}\right)^{\prime}=\alpha(\rho(p) h)^{\prime} \text { in } \Omega \\
p(a)=A, p(b)=B
\end{gathered}
$$


where $A, B$ are positive constants, $\alpha=6 \mu V_{1}>0$. As in the previous section $h$ is assumed to be a measurable function satisfying

$$
\begin{array}{r}
0<h_{1} \leqslant h(x) \leqslant h_{2} \text { a.e. } x \in \Omega \\
\left|h^{\prime}(x)\right| \leqslant H \text { a.e. } x \in \Omega
\end{array}
$$

for some constants $h_{1}, h_{2}, H$.

(We have denoted by ' the derivative $\frac{d}{d x}$ )

Moreover we will assume that $\rho$ satisfies (9). (Note that we do not assume $p$ is increasing, this will introduce some complications later). Under the assumptions $(9)(31)$ then it makes sense to look for a $p$ in $w^{1,1}(\Omega)$ as a solution to (29). (we refer the reader to [6] for the definition of these Sobolev spaces). Indeed, if $p \in W^{1,1}(\Omega)$, then $p$ is absolutely continuous on $\bar{\Omega}$ and thus $h^{3} \rho(p)$ is bounded. This implies that $h^{3} \rho(p) p^{1} \in L^{1}(\Omega)$ and (29) makes sense as an equality between two distributions.

In this context we can prove the following comparison result:

Theorem 2: Let $p_{i} \in W^{1,1}(\Omega) \quad(i=1,2)$ be positive functions satisfying:

$$
\begin{gathered}
\left(h^{3} \rho\left(p_{j}\right) p_{j}^{\prime}\right)^{\prime}=\alpha\left(\rho\left(p_{j}\right) h\right)^{\prime} \text { in } \Omega \\
p_{i}(a)=A_{i}, p_{j}(b)=B_{i}
\end{gathered}
$$

where $A_{j}, B_{i}$ are two positive constants. Assume that $(9)(31)$ nold then

$$
\begin{aligned}
& A_{1} \geqslant A_{2}, \quad B_{1} \geqslant B_{2} \Rightarrow p_{1} \geqslant p_{2} \quad \text { in } \Omega, \\
& A_{1}>A_{2}, \quad B_{1} \geqslant B_{2} \Rightarrow p_{1}>p_{2} \quad \text { in } \Omega .
\end{aligned}
$$

Proof: Assume that (35) does not hold. Then there exists $x_{0} \in(a, b)$ for which

$$
\left(p_{1}-p_{2}\right)\left(x_{0}\right)<0
$$

Let us denote by $\left(\alpha_{1}, \alpha_{2}\right)$ the connected component of $x_{0}$ in the set 


$$
\left[p_{1}-p_{2}<0\right]=\left\{x \in \Omega \mid\left(p_{1}-p_{2}\right)(x)<0\right\}
$$

Thanks to our assumptions $A_{1} \geqslant A_{2}, B_{1} \geqslant B_{2}$ we have

$$
\left(p_{1}-p_{2}\right)\left(\alpha_{1}\right)=\left(p_{1}-p_{2}\right)\left(\alpha_{2}\right)=0
$$

Moreover from (33) we deduce that there exists a constant $C$ such that

$$
c+h^{3}\left(\rho\left(p_{1}\right) p_{1}^{1}-\rho\left(p_{2}\right) p_{2}^{\prime}\right)=\alpha h\left(\rho\left(p_{1}\right)-\rho\left(p_{2}\right)\right) \text { in }\left(\alpha_{1}, \alpha_{2}\right) \text {. }
$$

Using (10) this reads

$$
C+h^{3}\left(R\left(p_{1}\right)-R\left(p_{2}\right)\right)^{\prime}=\alpha h\left(\rho\left(p_{1}\right)-\rho\left(p_{2}\right)\right) \text { in }\left(\alpha_{1}, \alpha_{2}\right)
$$

Dividing both sides of this equality by $h^{3}$ and integrating over $\left(\alpha_{1}, \alpha_{2}\right)$, we get

$$
\text { C } \int_{\alpha_{1}}^{\alpha_{2}} \frac{d x}{h^{3}}=\alpha \int_{\alpha_{1}}^{\alpha_{2}} \frac{\rho\left(p_{1}\right)-\rho\left(p_{2}\right)}{h^{2}} d x \leqslant 0 \text {. }
$$

If $\rho\left(p_{1}\right) \equiv \rho\left(p_{2}\right)$ on $\left(\alpha_{1}, \alpha_{2}\right)$ we obtain from (39)

$$
\rho\left(p_{1}\right) p_{1}^{\prime}=\rho\left(p_{2}\right) p_{2}^{\prime} \quad \text { on } \quad\left(\alpha_{1}, \alpha_{2}\right)
$$

and thus

$$
\left(p_{1}-p_{2}\right)^{\prime}=0 \quad \text { on }\left(\alpha_{1}, \alpha_{2}\right) \text {. }
$$

But this is clearly impossible (see (37)(38)). Thus from (40) we deduce that $c<0$. Then, from $(38)(39)$ we see that $\left(R\left(p_{1}\right)-R\left(p_{2}\right)\right)^{\prime}(x)>0$ for $x$ in $\Omega$ close to $\alpha_{1}$. So, $\left(R\left(p_{1}\right)-R\left(p_{2}\right)\right)(x)>0$ for $x$ in $\left(\alpha_{1}, \alpha_{2}\right)$ close to $\alpha_{1}$. But this contradicts the fact that $p_{1}-p_{2}<0$ on $\left(\alpha_{1}, \alpha_{2}\right)$ (Thanks to (9), $R$ is non decreasing) thus (37) cannot hold and this completes the proof of (35). To get (36) we note first that by $(35)$ we have $\left(p_{1}-p_{2}\right)(x) \geqslant 0$. Assume that for some $x_{0} \in \Omega$

$$
\left(p_{1}-p_{2}\right)(x)>0 \text { on }\left(a, x_{0}\right),\left(p_{1}-p_{2}\right)\left(x_{0}\right)=0 \text {. }
$$


Arguing as in (39) we have for some constant $C$

$$
C+h^{3}\left(R\left(p_{1}\right)-R\left(p_{2}\right)\right)^{\prime}=\alpha h\left(\rho\left(p_{1}\right)-\rho\left(p_{2}\right)\right) \text { in } \Omega \text {. }
$$

If $C \leqslant 0$ we deduce from the above equality that

$$
\left(R\left(p_{1}\right)-R\left(p_{2}\right)\right)^{\prime} \geqslant 0 \text { in }\left(a, x_{0}\right)
$$

thus $\left(R\left(p_{1}\right)-R\left(p_{2}\right)\right)\left(x_{0}\right)>0$ which contradicts $(41)$. If $c>0$, then for $x$ close to $x_{0}$

$$
\left(R\left(p_{1}\right)-R\left(p_{2}\right)\right)^{\prime}(x)<0
$$

and thus for $x>x_{0}$, $x$ close to $x_{0}$ we have $\left(R\left(p_{1}\right)-R\left(p_{2}\right)\right)(x)<0$ which contradicts (35). This completes the proof of the theorem.

As an obvious corollary of Theorem 2 we have

Theorem 3: Assume that (9)(31) hold. Then, there exists at most one positive solution $p \in W^{1,1}(\Omega)$ of $(29)(30)$.

Moreover:

Theorem 4: Assume that $(9)(31)$ nolds and that $p \in W^{1,1}(\Omega)$ is a positive solution of $(29)(30)$. Then:

$$
A>0, B \geqslant 0 \Rightarrow p>0 \text { on }(a, b) \text {. }
$$

Proof: It is enough to note that the function 0 is the solution of (29)(30) corresponding to $A=B=0$ and Theorem 4 is then a consequence of Theorem 2 applied with $p_{1}=p, p_{2}=0$.

Remark 3: If $A=0$ the following example (see Cimatti [5]) shows that (42) may fail. Take $\Omega=(0,1), \alpha=1, h \equiv 1, B=1 / 2, \rho(s)=s$. Then $p$ defined by

$$
\begin{aligned}
p(x)= & 0 \text { on }(0,1 / 2) \\
& x-1 / 2 \text { on }(1 / 2,1)
\end{aligned}
$$


is a function in $W^{1,1}(\Omega)$ satisfying $(29),(30)$. So, note that in this case $(29),(30)$ becomes a free boundary problem.

The next theorem combines the results of this section with those of the previous one. First let us set for $s \geqslant 0$

$$
\beta(s)=\alpha \rho\left(R^{-1}(s)\right)
$$

and assume that

$$
\exists c>0 \text { such that }|B(s)| \leqslant \frac{\sqrt{v h_{1}^{3}}}{2 h_{2}} s+c \quad \forall s>0
$$

where $\nu$ is the smallest eigenvalue of the problem (19) where $\Delta$ is replaced by $\frac{d^{2}}{d x^{2}}$

Then:

Theorem 5: Assume that $(9)(31)(43)$ hold. Then for $A, B>0$ there exists a unique positive solution $p \in W^{1,1}(\Omega)$ of $(29),(30)$. Moreover, $u=R(p)$ is the unique solution of the problem

$$
\begin{gathered}
u \in H^{l}(\Omega), u(a)=R(A), \quad u(b)=R(B) \\
\int_{\Omega} h^{3} u^{\prime} \cdot \xi^{\prime} d x=\alpha \int_{\Omega} h \rho \circ R^{-1}(u) . \xi^{\prime} d x \quad \forall \xi \in H_{0}^{l}(\Omega) .
\end{gathered}
$$

Proof: Let us extend $\beta$ for $s<0$ by the formula

$$
\beta(s)=\alpha \rho\left(R^{-1}(0)\right)=0
$$

Then, thanks to (43) one can apply Theorem 1 (this theorem has been established in two dimensions but it holds clearly without any change in the proof in dimension one) So, there exists a solution $u \in H^{1}(\Omega)$ of the problem

$$
\begin{aligned}
u(a) & =R(A), \quad u(b)=R(B) \\
\int_{\Omega} h^{3} u^{\prime} \cdot \xi^{\prime} d x & =\int_{\Omega} h \cdot \beta(u) \cdot \xi^{\prime} d x \quad \forall \xi \in H_{0}^{1}(\Omega) .
\end{aligned}
$$


We claim that $u$ is strictly positive. Indeed, if not, let $x_{0}$ be the point of $(a, b)$ such that

$$
u\left(x_{0}\right)=0, u(x)>0 \text { on }\left(x_{0}, b\right)
$$

From (47) we deduce that for some constant $C$

$$
C+h^{3} u^{\prime}=h \beta(u) \text { in } \Omega \text {. }
$$

Dividing by $h^{3}$ and integrating on $\left(a, x_{0}\right)$ we get

$$
\text { c } \int_{a}^{x_{0}} \frac{d x}{h^{3}}-u(a)=\int_{a}^{x_{0}} \frac{\beta(u)}{h^{2}} d x
$$

and thus $c>0$. Now, from $(49)$, since $B(0)=0$, we deduce that $u^{\prime}(x)<0$ for $x \in\left(x_{0}, b\right), x$ close to $x_{0}$. This is a contradiction to (48). Then we can define

$$
p=R^{-1}(u)
$$

Since $u$ is strictly positive (the derivative of $R^{-1}$ has a singularity at 0 ) the function $p$ is clearly in $H^{1}(\Omega)$ and satisfies $(29)(30)$. This completes the proof thanks to theorem 2.

Remark 4: Under the assumptions of Theorem 5 if $\rho$ and $h$ are "smooth" then $\mathrm{p}$ is "smooth" and thus a classical solution of (29)(30) (This can be seen by a usual bootstrap argument). Note that in fact, up to now, (32) has not been used. Now, if (32) holds, and $\rho$ is Lipschitz continuous, then under the assumptions of theorem $5, p \in W^{2, \infty}(\Omega)$ and in particular $p$ is a function of class $C^{1}$ on $\bar{\Omega}$.

In the rest of this section we will describe more precisely the profile of the pressure $p$ solution of $(29)(30)$. We are going to restrict ourselves to the case $A=B$ and so we consider $p \in W^{1,1}(\Omega)$, solution of the problem

$$
\begin{gathered}
\left(h^{3} \rho(p) p^{\prime}\right)^{\prime}=\alpha(\rho(p) h)^{\prime} \text { in } \Omega=(a, b) \\
p(a)=p(b)=P_{0}>0
\end{gathered}
$$


where $P_{0}$ is some positive constant.

Roughly speaking we are going to prove that for $h$ as in the picture, $p$ is as below.

$n:$

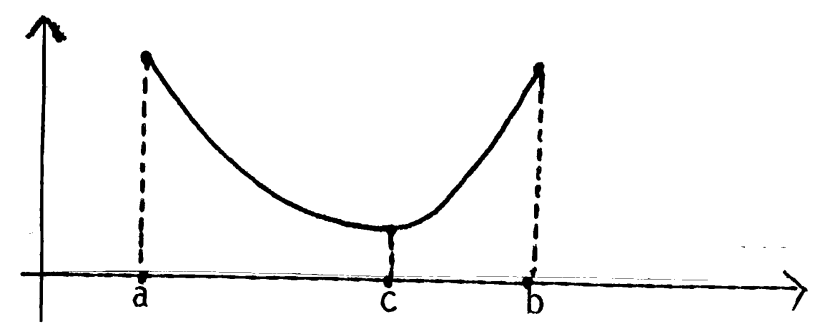

$p:$

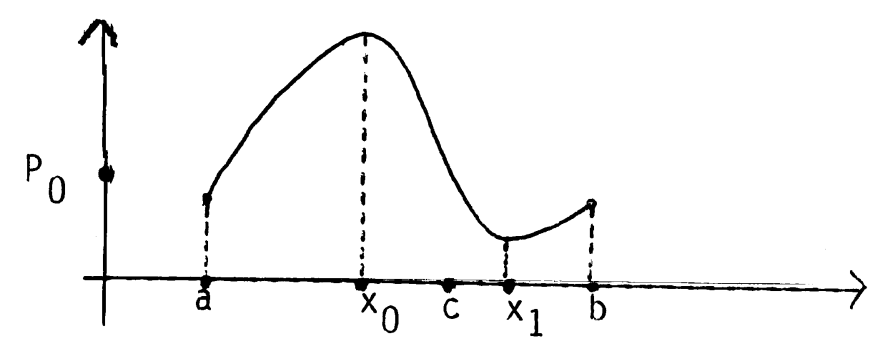

In addition to (31)(32) we will assume provisionally

$$
\begin{gathered}
h \text { is a function of class } c^{1} \text { near a, } \\
h(a)=h(b), \quad h^{\prime}(a)<0,
\end{gathered}
$$

$\exists c \in(a, b)$ such that $h$ is decreasing on $(a, c)$ increasing on $(c, b)$.

Moreover we will assume on $\rho$ that

$$
\rho \text { is a locally Lipschitz continuous function on }(0,+\infty) \text {. }
$$

Under these conditions we first prove:

Lemma 1: Assume that $(9)(31)(32)(52)-(55)$ hold then if $p$ is the solution of $(50)(51)$ we have:

$$
p^{\prime}(a)=p^{\prime}(b) \geqslant 0
$$

Proof: First note that thanks to our assumptions $p$ is a function of class $c^{l}$ on $\Omega$ (see Remark 4). Next, thanks to (50), we have in $\bar{\Omega}$ for some constant $C$ 


$$
h^{3} \rho(p) p^{\prime}+C=\alpha \rho(p) h \text {. }
$$

This relation at the points a,b implies (see (51)(53))

$$
p^{\prime}(a)=p^{\prime}(b)
$$

Next, suppose that $p^{\prime}(a)<0$. Then $p$ is decreasing around $a$. Let $x_{0}$ be the first point such that $p^{\prime}\left(x_{0}\right)=0$ (i.e. $p^{\prime}(x)<0$ on $\left[a, x_{0}\right)$ ). Clearly such $a$ point exists since $p(a)=p(b)$. From (57) we get

$$
C=\alpha \rho(p)\left(x_{0}\right) h\left(x_{0}\right) .
$$

Since $p^{\prime}(x)<0$ on $\left[a, x_{0}\right)$, and by $(53)(54)$ :

$$
p\left(x_{0}\right)<p(a) \quad h\left(x_{0}\right)<h(a) .
$$

Hence

$$
C=\alpha \rho(p)\left(x_{0}\right) h\left(x_{0}\right)<\alpha \rho(p)(a) h(a)=h^{3}(a) \rho(p(a)) p^{\prime}(a)+C<C .
$$

This is impossible, and thus (56) holds.

Next we have:

Lemma 2: Assume that $(9)(31)(32)(52)-(55)$ hold then if $p$ is the solution of (50)(51) we have:

$$
p^{\prime}(a)=p^{\prime}(b)>0
$$

Proof: Thanks to (56) if (58) does not hold we would have

$$
p^{\prime}(a)=0 \text {. }
$$

Thus by (57)

$$
C=\alpha \rho(p)(a) h(a)
$$

and we have

$$
h^{3} \rho(p)(x) p^{\prime}(x)=\alpha \rho(p)(x) h(x)-\alpha \rho(p)(a) h(a) .
$$


This implies for $x>a$

$$
\frac{h^{3} \rho(p)(x) p^{\prime}(x)}{x-a}=\alpha\left(\frac{\rho(p)(x)-\rho(p)(a)}{x-a}\right) h(x)+\alpha \rho(p)(a)\left(\frac{h(x)-h(a)}{x-a}\right) \text {. }
$$

Letting $x$ go to a, from (55) we deduce that

$$
\left|\frac{p(p(x))-p(p(a))}{x-a}\right| \leqslant c\left|\frac{p(x)-p(a)}{x-a}\right| \rightarrow c\left|p^{\prime}(a)\right|=0
$$

Thus, when $x$ goes to $a$, the right side of $(60)$ goes to

$$
\alpha \rho(p(a)) h^{\prime}(a)<0
$$

(see (53)). So, for $x$ close to a we deduce from (60) that

$$
p^{\prime}(x)<0
$$

As in the preceeding lemma let $x_{0}$ be the first point such that

$$
p^{\prime}\left(x_{0}\right)=0 \quad p^{\prime}(x)<0 \text { on }\left(a, x_{0}\right) \text {. }
$$

we have

$$
p\left(x_{0}\right)<p(a), \quad h\left(x_{0}\right)<h(a)
$$

and at the same time (see (57)(59))

$$
C=\alpha \rho(p)\left(x_{0}\right) h\left(x_{0}\right)=\alpha \rho(p)(a) h(a)
$$

which is impossible. Thus (59) cannot hold and we have (58).

Lemma 3: Assume that $(9)(31)(32)(52)-(55)$ hold. Let $p$ be the solution of $(50)(51)$

(i) If $a \leqslant \alpha_{1}<\alpha_{2} \leqslant c$ we cannot have $p^{\prime}\left(\alpha_{1}\right)=p^{\prime}\left(\alpha_{2}\right)=0, p^{\prime} \leqslant 0$ on $\left(\alpha_{1} \cdot \alpha_{2}\right)$.

(ii) If $c \leqslant \alpha_{1}<\alpha_{2} \leqslant b$ we cannot have $p^{\prime}\left(\alpha_{1}\right)=p^{\prime}\left(\alpha_{2}\right)=0, p^{\prime} \geqslant 0$ on $\left(\alpha_{1}, \alpha_{2}\right)$.

Proof. (i) If $p^{\prime}\left(\alpha_{1}\right)=p^{\prime}\left(\alpha_{2}\right)=0$ we have (see (57)) 


$$
C=\alpha \rho(p)\left(\alpha_{1}\right) h\left(\alpha_{1}\right)=\alpha \rho(p)\left(\alpha_{2}\right) h\left(\alpha_{2}\right) \text {. }
$$

Now since $p$ is non decreasing and thanks to (54):

$$
\rho(p)\left(\alpha_{1}\right) \geqslant \rho(p)\left(\alpha_{2}\right), h\left(\alpha_{1}\right)>h\left(\alpha_{2}\right)
$$

and (61) is impossible.

(ii) Same proof.

We are now able to describe the profile of the pressure $p$. We have:

Theorem 6: Assume that $(9)(31)(32)(52)$ - (55) hold. Then if $p$ is the solution of $(50)(51)$ there exist $x_{0} \in(a, c], x_{1} \in(c, b)$ such that:

(i) $p$ is non decreasing on $\left(a, x_{0}\right)$ and increasing on $\left(x_{1}, b\right)$,

(ii) $p$ is non increasing on $\left(x_{0}, x_{1}\right)$.

Proof: Set

$$
x_{0}=\operatorname{Sup}\left\{x \mid P^{\prime}\left(x^{\prime}\right) \geqslant 0 \quad \forall \quad x^{\prime} \in(a, x)\right\}
$$

We have $p^{\prime}\left(x_{0}\right)=0$. (If not then $x_{0}<b$ and one has a contradiction with the definition of $x_{0}$ ). Moreover $x_{0} \leqslant c$. If not from (57) we deduce

$$
h^{3} \rho(p) p^{\prime}=\alpha h_{\rho}(p)-C=\alpha h \rho(p)-h\left(x_{0}\right) \rho(p)\left(x_{0}\right) \text { on }\left(a, x_{0}\right) \text {. }
$$

Noting that

$$
p(c) \leqslant p\left(x_{0}\right) \quad h(c)<h\left(x_{0}\right)
$$

we obtain $p^{\prime}(c)<0$ and a contradiction with the definition of $x_{0}$.

Set

$$
x_{1}=\operatorname{Inf}\left\{x \mid p^{\prime}\left(x^{\prime}\right) \geqslant 0 \forall x^{\prime} \in(x, b)\right\}
$$

First we note that $x_{1}>x_{0}$ - otherwise $p$ would be non decreasing on ( $\left.a, b\right)$ which is impossible since $p$ is not constant (see lemma 2). 
Next we claim that $p^{\prime}<0$ on $\left(x_{0}, c\right]$. Indeed if not there exists $\delta \in\left(x_{0}, c\right]$ such that $p^{\prime}(\delta) \geqslant 0$. By definition of $x_{0}$, there exists $\gamma \in\left(x_{0}, \delta\right)$ such that $\mathrm{p}^{\prime}(\gamma)<0$. If $\left(\alpha_{1}, \alpha_{2}\right)$ is the connected component of $\gamma$ in the set $\left\{x \in\left(x_{0}, \delta\right) \mid p^{\prime}(x)<0\right\}$, then we have a contradiction with (i) of Lemma 2. The above implies also that $x_{1} \in(c, b)$.

Next we prove that $p^{\prime}$ is non positive on $\left(c, x_{1}\right)$. If not there will exist $\tau \in\left(c, x_{1}\right)$ such that $p^{\prime}(\tau)>0$. Considering $\left(\alpha_{1}, \alpha_{2}\right)$ as to be the connected component of $\tau$ in the set $\left\{x \in\left(c, x_{1}\right) \mid p^{\prime}(x)>0\right\}$ we are in contradiction with ( $i i)$ of lemma 2. Now the fact that $p^{\prime}>0$ on $(x, b)$ results easily from (ii) of Lemma 3. This completes the proof of the theorem.

Remark 5: Assume that $\rho$ and $h$ are smooth, more precisely of class $c^{l}$, then it is easy to check that $p$ is of class $c^{2}$. Moreover one has in $(c, b)$

$$
h^{3} \rho(p) p^{\prime \prime}+h^{3} \rho^{\prime}(p) p^{\prime} p^{\prime}-\alpha h \rho^{\prime}(p) p^{\prime}=\alpha h^{\prime} \rho(p) \geqslant 0 .
$$

By the maximum principle (see [9]) if the maximum of $p$ is achieved at $c$ we have

$$
p^{\prime}(c)<0
$$

So in this case one has necessarily $x_{0}<c$. (If not we would have $p^{\prime}\left(x_{0}\right)=p^{\prime}(c)=0$ which contradicts the above inequality).

For a similar reason:

$$
p^{\prime}(x)<0 \text { on }\left(c, x_{1}\right), p^{\prime}(x)>0 \text { on }\left(a, x_{0}\right)
$$

and thus (see the proof of the above theorem)

$p$ is increasing on $\left(a, x_{0}\right)$ and $\left(x_{1}, b\right)$

$p$ is decreasing on $\left(x_{0}, x_{1}\right)$.

Indeed, if for some $d \in\left(a, x_{0}\right)$ we have

$$
p^{\prime}(d)=0
$$


then $d$ is a minimum for $p$ on $\left(d, x_{0}\right)$ and

$$
h^{3} \rho(p) p^{\prime \prime}+h^{3} \rho^{\prime}(p) p^{\prime} p^{\prime}-\alpha h \rho^{\prime}(p) p^{\prime}=\alpha h^{\prime} \rho(p) \leqslant 0 \text { on }\left(d, x_{0}\right) \text {. }
$$

Then by the maximum principle we have $p^{\prime}(d)>0$, which contradicts (63). The proof of $p^{\prime}(x)<0$ on $\left(c, x_{1}\right)$ is the same.

In the next theorem we relax the assumptions (52)(53).

We have:

Theorem 7: Assume that $(9)(31)(32)(54)(55)$ hold. Then if $p$ is the solution of $(50)(51)$ there exist $x_{0} \in[a, c]$ and $x_{1} \in(c, b]$ such that:

(i) $p$ is nondecreasing on $\left(a, x_{0}\right)$ and $\left(x_{1} b\right)$,

(ii) $p$ is non increasing on $\left(x_{0}, x_{1}\right)$.

Proof: If $h$ satisfy (54) then we can find a sequence $h_{n}$ of functions satisfying $(31)(32)(52)(53)(54)$ and such that

$$
\begin{aligned}
& h_{n}(x)+h(x) \text { a.e. on } \Omega, \\
& h_{n}(x) \rightarrow h(x) \text { in } L^{2}(\Omega) .
\end{aligned}
$$

Indeed the picture below gives the recipe of how to construct $h_{n}$ (the doted function)

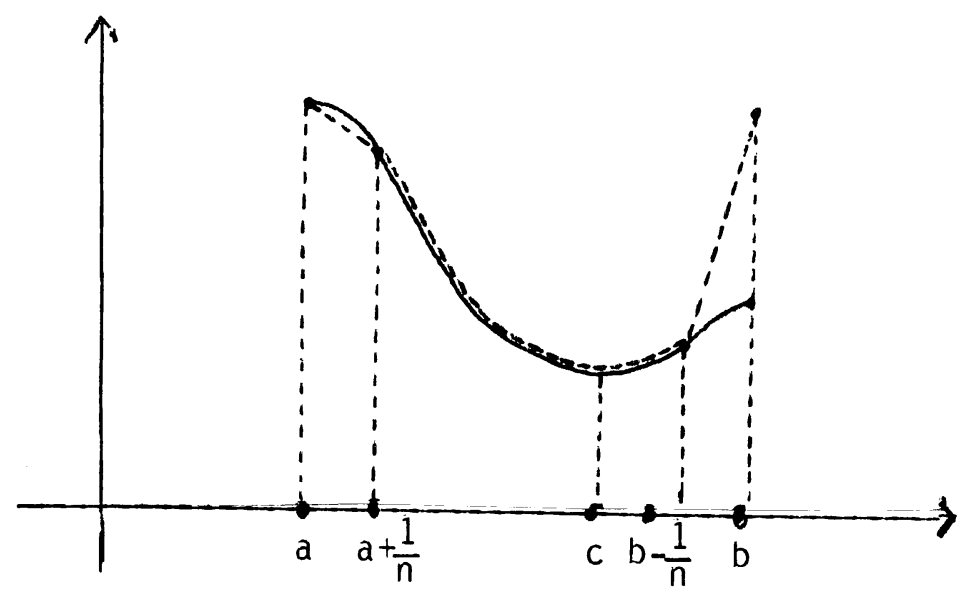

Then, we consider the solution $u_{n}$ of $(44)(45)$ with $h=h_{n}, A=B=P_{0}$. From 
theorem 6 we deduce that there exists $x_{0}^{n} \in(a, c], x_{1}^{n} \in(c, b)$ such that $u_{n}$ is non decreasing on $\left(a, x_{0}^{n}\right)$ and $\left(x_{1}^{n}, b\right)$ and $u_{n}$ is non increasing on $\left(x_{0}^{n}, x_{1}^{n}\right)$. Extract a subsequence such that

$$
\begin{aligned}
& x_{0}^{n} \rightarrow x_{0} \in[a, c] \\
& x_{1}^{n} \rightarrow x_{1} \in[c, b] \\
& u_{n} \rightarrow u \text { in } L^{2}(\Omega), u_{n}^{\prime} \rightarrow u^{\prime} \text { in } L^{2}, u_{n} \rightarrow u_{0} \text { a.e. }
\end{aligned}
$$

(Note that thanks to (31) $u_{n}$ is bounded in $H^{1}(\Omega)$.)

then by standard arguments we can easily prove that $u=R(p)$ (see the proof of theorem 1).

It results that:

$u$ and thus $p$ is non decreasing on $\left(a, x_{0}\right),\left(x_{1}, b\right)$,

$u$ and $p$ are non increasing on $\left(x_{0}, x_{1}\right)$.

(one of these intervals could be empty, but not two of them since $p=C s t$ is not a solution of $(50)(51)$ under the assumption (54)).

Remark 6: Clearly by similar arguments the theorem 7 holds if we assume only $h$ non increasing on $(a, c), h$ non decreasing on $(c, b)$. In the case where $c=a$ or $c=b$ we get that $x_{0}=a$ or $x_{1}=b$ and a result can be stated in both cases. We leave to the reader the elaboration of a precise statement. Note that in each of the above cases the shape of $p$ can be described more precisely by the techniques of Lemma 2 or Remark 5.

\section{Back to Higher Dimensions}

\subsection{Uniqueness and positivity of the solutions}

In this section, in addition to (17) we will assume that the function $\beta$ defined by (14) and extended by 0 for $s<0$ satisfies for some $\tau>0$ : 


$$
\begin{aligned}
& \beta \text { is one-to one from }(0 . \tau) \text { onto }(0, \beta(\tau)) \text {, } \\
& \beta^{-1} \text { is a } c^{2} \text { function on }(0, \beta(\tau)) \text { such that } \\
& \lim _{x \rightarrow 0} \frac{d}{d x} \beta^{-1}(x)=\lim _{x \rightarrow 0} \times \frac{d^{2}}{d x^{2}} B^{-1}(x)=0 .
\end{aligned}
$$

For $\delta \geqslant 0$ we set:

$$
\omega_{\delta}(t)=\operatorname{Sup}_{\substack{x, y \in(\delta,+\infty) \\|x-y| \leqslant t}}|\beta(x)-\beta(y)|
$$

and we also assume:

$$
\int_{0} \frac{d t}{\omega_{\delta}^{2}(t)}=+\infty \quad \forall \delta>0
$$

Remark 7: It is easy to check that all the above assumptions hold for instance for a sinooth $\rho$ given by (20) near 0 . (Note that (67) holds if $B$ is Hölder continuous with exponent $\alpha \geqslant 1 / 2$ on $(0,+\infty))$

Moreover, if $\phi \in H^{1}(\Omega)$, we will suppose that for some positive constant c:

$$
\phi \geqslant c>0 \text { on } \Gamma
$$

and in addition to (3) we will also assume that

$$
n \in w^{2,1}(\Omega)
$$

Under the above assumptions we are going to prove that there is at most one solution $u$ to (22)(23). Moreover this solution is strictly positive and, if our data are smooth, is a classical solution to (15)(16). Note that the strict positivity of $u$ is not a trivial consequence of the maximum principle.

Let $k$ be a smooth, bounded, strictly positive function such that

$$
\underline{V} \cdot \nabla k \leqslant k<0
$$

where $K$ is a constant. For $\varepsilon>0$ small enough we have clearly 


$$
0<\varepsilon \frac{\dot{k}}{h} \leqslant \beta(\tau)
$$

and we can define (see (64))

$$
u_{\varepsilon}=\beta^{-1}\left(\varepsilon \frac{\dot{k}}{h}\right)
$$

We will say that $v \in H^{1}(\Omega)$ is a subsolution of (22)(23) iff:

$$
\begin{gathered}
(v-\phi)^{+} \in H_{0}^{l}(\Omega), \\
\int_{\Omega} h^{3} \nabla v \cdot \nabla \xi-h_{\beta}(v) \underline{v} \cdot \nabla \xi d x \leqslant 0 \quad \forall \xi \in H_{0}^{1}(\Omega), \quad \xi \geqslant 0 \text { a.e. in } \Omega,
\end{gathered}
$$

where ()$^{+}$denotes the positive part of a function.

Then we have:

Proposition 1: For $\varepsilon$ small enough $u_{\varepsilon}$ is a subsolution of (22)(23).

Proof: Clearly $u_{\varepsilon} \in H^{1}(\Omega)$ and for $\varepsilon$ small enough $\left(\beta^{-1}(x) \rightarrow 0\right.$ when $\left.x \rightarrow 0\right)$

$$
u_{\varepsilon}=\beta^{-1}\left(\frac{\varepsilon k}{h}\right) \leqslant c \leqslant \phi .
$$

Hence (72) holds. Next, we have for $\xi \in H_{0}^{1}(\Omega)$.

$$
\begin{aligned}
& \int_{\Omega} h^{3} \nabla u_{\varepsilon} \cdot \nabla \xi-h_{\beta}\left(u_{\varepsilon}\right) \underline{V} \cdot \nabla \xi d x=\int_{\Omega} h^{3} \nabla \beta^{-1}\left(\varepsilon \frac{k}{h}\right) \cdot \nabla \xi-h \beta\left(\beta^{-1}\left(\varepsilon \frac{k}{h}\right)\right) \underline{V} \cdot \nabla \xi d x \\
& =\int_{\Omega}\left[-\nabla \cdot\left(h^{3} \nabla \beta^{-1}\left(\varepsilon \frac{k}{h}\right)\right)+\varepsilon \underline{V} \cdot \nabla k\right] \cdot \xi d x \\
& =\varepsilon \int_{\Omega}\left[-\nabla \cdot\left(h^{3}\left(\beta^{-1}\right)^{\prime}\left(\varepsilon \frac{k}{h}\right) \cdot \nabla\left(\frac{k}{h}\right)\right)+\underline{V} \cdot \nabla k\right] \cdot \xi d x \\
& =\varepsilon \int_{\Omega}\left[-\nabla \cdot\left(h^{3} \nabla\left(\frac{k}{h}\right)\right) \cdot\left(\beta^{-1}\right)^{\prime}\left(\varepsilon \frac{k}{h}\right)-h^{3} \varepsilon\left(\beta^{-1}\right)^{\prime \prime}\left(\frac{\varepsilon k}{h}\right)\left|\nabla\left(\frac{k}{h}\right)\right|^{2}+\underline{V} \cdot \nabla k\right] \cdot \xi d x .
\end{aligned}
$$

Thanks to $(65)$, when $\varepsilon$ goes to 0 the integrand goes to $\underline{V} \cdot \nabla k$, and thus (see 
(70)) we have for $\varepsilon$ small enough, $\xi \in H_{0}^{l}(\Omega), \xi \geqslant 0$,

$$
\int_{\Omega}\left(h^{3} \nabla u_{\varepsilon} \cdot \nabla \xi-h \beta\left(u_{\varepsilon}\right) \underline{v} \cdot \nabla \xi\right) d x \leqslant 0
$$

This concludes the proof of the proposition.

Next we have:

Theorem 8: Let $u$ be a solution of (22)(23) and $v$ a subsolution of (22)(23) such that for some

$\delta>0$ we have

$$
v \geqslant 2 \delta>0 \text { a.e. in } \Omega \text {. }
$$

Then

$$
v \leqslant u \text { a.e. in } \Omega
$$

Proof: First we prove that for all $\zeta \in C^{\infty}(\Omega), \zeta \geqslant 0$ :

$$
\int_{[v-u>0]} h^{3} \nabla(v-u) \cdot \nabla \zeta-h(\beta(v)-\beta(u)) \underline{v} \cdot \nabla \zeta d x \leqslant 0
$$

where

$$
[v-u>0]=\{x \in \Omega \mid(v-u)(x)>0\} .
$$

To do this we use the technique of [2]. For $\delta$ satisfying (74) we have:

$$
\int_{0} \frac{d t}{\omega_{\delta}(t)^{2}}=
$$

So, for $\varepsilon>0$, there exists a number $\ell(\varepsilon)<\varepsilon$ such that

$$
\int_{\ell(\varepsilon)}^{\varepsilon} \frac{d t}{\omega_{\delta}(t)^{2}}=1 .
$$

Define the function $F_{\varepsilon}(t)$ by

$$
\begin{array}{ll}
F_{\varepsilon}(t)=\int_{l(\varepsilon)}^{t} d t / \omega_{\delta}(t)^{2} & \text { if } t \leqslant \ell(\varepsilon) \\
1 & \text { if } \ell(\varepsilon) \leqslant t \leqslant \varepsilon
\end{array}
$$


Clearly the function $F_{\varepsilon}$ is Lipschitz continuous and for $\zeta \in C^{\infty}(\bar{\Omega})$, ${ }^{\mathrm{F}}{ }_{\varepsilon}(v-u) \in H_{0}^{l}(\Omega)$. Moreover by definition of $u$ and $v$, we have:

$$
\begin{aligned}
& \int_{\Omega} h^{3} \nabla u \cdot \nabla \xi-h \beta(u) \underline{V} \cdot \nabla \xi d x=0 \quad \forall \xi \in H_{0}^{l}(\Omega), \\
& \int_{\Omega} h^{3} \nabla v \cdot \nabla \xi-h \beta(v) \underline{V} \cdot \nabla \xi d x \leqslant 0 \quad \forall \xi \in H_{0}^{1}(\Omega), \quad \xi \geqslant 0,
\end{aligned}
$$

and thus

$$
\int_{\Omega} h^{3} \nabla(v-u) \cdot \nabla \xi-h(\beta(v)-\beta(u)) \underline{v} \cdot \xi \leqslant 0 \quad \forall \xi \in H_{0}^{1}(\Omega), \quad \xi \geqslant 0 .
$$

If $\zeta \geqslant 0$, it is clear that $\xi=\zeta_{\varepsilon} F_{\varepsilon}(v-u)$ is a suitable test function for the above inequality. So we obtain:

$$
\begin{aligned}
& I_{\varepsilon}=\int_{\Omega}\left[h^{3} \nabla(v-u) \cdot \nabla \zeta-h(\beta(v)-\beta(u)) \underline{V} \cdot \nabla \zeta\right] F_{\varepsilon}(v-u) d x \leqslant \\
&-\int_{\Omega}\left[h^{3} \nabla(v-u) \cdot \nabla F_{\varepsilon}(v-u)-h(\beta(v)-\beta(u)) \underline{V} \cdot \nabla F_{\varepsilon}(v-u)\right] \zeta d x \\
& \leqslant-h_{1}^{3} \int_{\Omega}|\nabla(v-u)|^{2} F_{\varepsilon}^{\prime}(v-u) \cdot \zeta d x+h_{2}|\underline{V}| \int_{\Omega}|\beta(v)-\beta(u)||\nabla(v-u)| F_{\varepsilon}^{\prime}(v-u) \zeta d x .
\end{aligned}
$$

Then using the inequality

$$
x y \leqslant \frac{1}{2}\left(\frac{h_{2}|\underline{V}|}{h_{1}^{3}} x^{2}+\frac{h_{1}^{3}}{h_{2} \underline{I V T}} y^{2}\right) \quad \forall x, y \in \mathbb{R}
$$

we get:

$$
\begin{aligned}
h_{2}|\underline{v}| \int_{\Omega} \mid \beta(v) & -\left.\beta(u)|| \nabla(v-u)\left|F_{\varepsilon}^{\prime}(v-u) \zeta d x \leqslant \frac{h_{1}^{3}}{2} \int_{\Omega}\right| \nabla(v-u)\right|^{2} F_{\varepsilon}^{\prime}(v-u) \zeta d x \\
& +\frac{h_{2}^{2}|v|^{2}}{2 h_{1}^{3}} \int_{\Omega}(\beta(v)-\beta(u))^{2} F_{\varepsilon}^{\prime}(v-u) \zeta d x .
\end{aligned}
$$

Combining this inequality and (78), and noting that $F_{\varepsilon}^{\prime} \geqslant 0$ we obtain: 


$$
I_{\varepsilon} \leqslant \frac{h_{2}^{2}|v|^{2}}{2 h_{1}^{3}} \int_{\Omega}(\beta(v)-\beta(u))^{2} F_{\varepsilon}^{\prime}(v-u) \zeta d x .
$$

By (77) this leads to

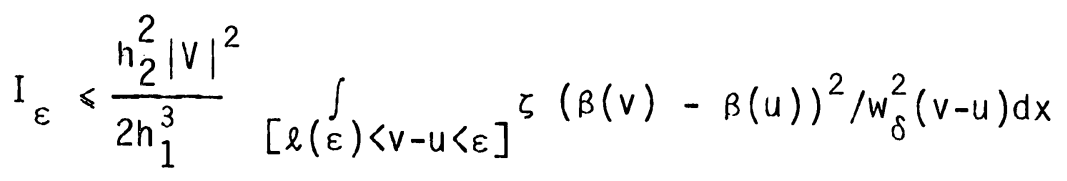

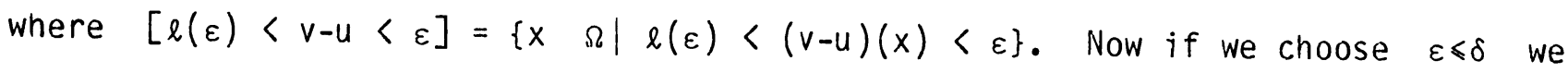
have on this set

$$
u>v-\varepsilon \geqslant 2 \delta-\varepsilon \geqslant \delta
$$

and by (66) we deduce that

$$
(\beta(v)-\beta(u))^{2} \leqslant \omega_{\delta}^{2}(v-u)
$$

So (79) reduces to:

$$
I_{\varepsilon} \leqslant \frac{h_{2}^{2}|V|^{2}}{2 h_{1}^{3}}\{\ell(\varepsilon)<v-u<\varepsilon]^{\zeta d x}
$$

and (76) follows by letting $\varepsilon$ go to zero, since $I_{\varepsilon}$ goes to the left hand side of $(76)$.

$$
\begin{aligned}
& \text { Next if } \underline{V}=\left(v_{1}, v_{2}\right) \text { we choose in }(76) \\
& \qquad=M-\exp \left[s\left(v_{2} x-v_{1} y\right)\right]
\end{aligned}
$$

where $M$ is a constant choosen large enough so that $\zeta \geqslant 0$ in $\Omega$, and $s$ is a constant to be chosen later. Since $\underline{V} \cdot \nabla \zeta \equiv 0$, we get

$$
\int_{[v-u>0]} h^{3} \nabla(v-u)^{+} \nabla \zeta \quad d x=\int_{\Omega} h^{3} \nabla(v-u)^{+} \cdot \nabla \zeta d x \leqslant 0 \quad .
$$

Integrating by parts leads to:

$$
\int_{\Omega}(v-u)^{+} \cdot-\nabla \cdot\left(h^{3} \nabla \zeta\right) d x \leqslant 0
$$

An easy computation shows that $s$ can be selected large enough so that 
and thus we have $(v-u)^{+}=0$ a.e. in $\Omega$ which is (75)

As a corollary of the previous theorem we have:

Theorem 9: Assume that $(3)(4)(17)(64)(65)(67)(68)$ hold. Then there exists at most one positive solution to (22)(23). Moreover this solution is strictly positive in $\Omega$. If in addition the data $h, \rho, \phi, \Omega$ are "smooth", then $u$ is smooth and $p=R^{-1}(u)$ is the classical solution of $(7)(8)$, with $P_{a}=R^{-1}(\phi)$.

Proof: Let $u, v$ be two solutions of (22)(23). Combining proposition 1 and theorem 8 we have for some $\delta>0$ and $\varepsilon$ small enough.

$$
u, v \geqslant u_{\varepsilon} \geqslant 2 \delta>0
$$

Then applying Theorem 8 to $u, v$ and $v, u$ (note that a solution is a subsolution) we get

$$
u \geqslant v \quad v \geqslant u \quad \text {. }
$$

Hence (22)(23) has at most one solution which is strictly positive on $\bar{\Omega}$. By a bootstrap argument we obtain easily that $u$ is smooth and the theorem follows.

Remark 8: If instead of (67) we assume

$$
\int_{0} \frac{d t}{\omega_{0}(t)^{2}}=+\infty
$$

than we can prove - assuming only that $\phi \geqslant 0$ on $\partial \Omega$ - that the solution of (22)(23) is unique. The proof goes like the proof of Theorem 8 (see [2]). However (80) does not hold when $\rho$ is given for example by (20) with $\alpha<1$. But we have proven that when (68) holds the solution $u$ stays away from the singularity 0 and in this case we still have uniqueness. The problem of uniqueness for the solution to (22)(23) when (67) and (68) fail remains open. As we mentioned already in the previous section in this case the problem becomes a free boundary problem. 


\subsection{Shape of the solution}

As a relevant physical case we are going to suppose in this section that $\phi$ and thus $p$ - are constant on the boundary of $\Omega$. Moreover we will assume that $\underline{V}=\left(V_{1}, 0\right), \quad h$ and $\rho$ are smooth, and so we will consider $p$ to be a smooth solution of the problem

$$
\begin{gathered}
\nabla \cdot\left(h^{3} \rho(p) \nabla p\right)=\alpha \frac{\partial}{\partial x}(h \rho(p)) \text { in } \Omega \\
p=p_{0}>0 \text { on } \partial \Omega
\end{gathered}
$$

where $p_{0}$ is some positive constant and $\alpha$ is given (see (6)) by:

$$
\alpha=\sigma_{\mu} V_{1}>0
$$

Denote by

$$
n=\left(n_{x}, n_{y}\right)
$$

the outward normal to $\Omega$.

Then we have:

Theorem 10: Assume that

$$
\partial_{\Omega} h n_{x} d \sigma \leqslant 0
$$

where $d \sigma$ denotes the surface measure on $\partial \Omega$. Moreover assume that

$$
\exists x_{0} \in \partial \Omega \text { where } \frac{\partial h}{\partial x}\left(x_{0}\right)>0
$$

then $p$ achieves a maximum strictly greater than $p_{0}$ inside $\Omega$.

Proof: If not $p \leqslant p_{0}$ in $\Omega$. This implies $\frac{\partial p}{\partial n} \geqslant 0$. Now around $x_{0}$ we have by $(84)$ :

$$
\nabla \cdot\left(h^{3} \rho(p) \nabla p\right)-\alpha h \rho^{\prime}(p) \frac{\partial p}{\partial x}=\alpha \rho(p) \frac{\partial h}{\partial x}>0 .
$$


So if we assume $p<p_{0}$ around $x_{0}$ by the strong maximum principle we have

$$
\frac{\partial p}{\partial n}\left(x_{0}\right)>0
$$

Integrating (81) over $\Omega$ and applying Green's formula we get

$$
\int_{\partial \Omega} h^{3} \rho\left(p_{0}\right) \frac{\partial p}{\partial n} d \sigma=\alpha \int_{\partial \Omega} h \rho\left(p_{0}\right) n_{x} d \sigma
$$

Hence dividing by the constant $\rho\left(p_{0}\right)$, we obtain

$$
\alpha \int_{\partial \Omega} h n_{x} d \sigma=\int_{\partial \Omega} h^{3} \frac{\partial p}{\partial n} d \sigma>0
$$

(since $\frac{\partial p}{\partial n} \geqslant 0$ on $\partial \Omega$ and thanks to (85)). But this contradicts (83) and thus we cannot have $\mathrm{p}<\mathrm{p}_{0}$ around $\mathrm{x}_{0}$. So for any neighborhood of $\mathrm{x}_{0}$ in $\Omega$ there exists a point in this neighborhood where $p \geqslant p_{0}$. Now if $p \leqslant p_{0}$ then we have found a point where $p$ achieves its maximum inside $\Omega$. According to the strong maximum principle this implies that $p \equiv p_{0}$ in a neighborhood of this point, but by (81) we would have

$$
\alpha \frac{\partial h}{\partial x} \equiv 0
$$

around this point and this contradicts (84) and completes the proof.

With exactly the same proof as in the previous theorem we have

Theorem 11: Assume that

$$
\int_{\partial \Omega} h n_{x} d \sigma \geqslant 0
$$

and that

$$
\exists x_{1} \in \partial \Omega \text { such that } \frac{\partial h}{\partial x}\left(x_{1}\right)<0
$$

then $p$ achieves a minimum strictly less than $p_{0}$ inside $\Omega$. 
Combining Theorem 10 and Theorem 11 we have the following result which generalizes theorem 6 to more than one dimension:

Theorem 12: Assume that (84)(88) hold and that

$$
\int_{\partial \Omega} h n_{x} d \sigma=0
$$

Then $p$ achieves a maximum strictly greater than $p_{0}$ in $\Omega$ and a minimum strictly less than $p_{0}$ in $\Omega$.

Remark 9: If we assume that $p$ is strictly positive, then we can get some more information about the shape of $p$ by noting that $p$ can achieve its maximum only in the region:

$$
\left[\frac{\partial h}{\partial x} \leqslant 0\right]=\left\{(x, y) \in \Omega \mid \frac{\partial h}{\partial x}(x, y) \leqslant 0\right\} .
$$

Indeed on the complement of this set in $\Omega$ we have by (81)

$$
\nabla \cdot\left(h^{3} \rho(p) \nabla p\right)-\alpha h \rho^{\prime}(p) \frac{\partial p}{\partial x}=\alpha \frac{\partial h}{\partial x} \rho(p)>0
$$

and by the maximum principle if $p$ achieves a maximum inside we have $p=C=c s t$. But then by (81) we get

$$
0=\alpha \rho(C) \frac{\partial h}{\partial x}>0
$$

which is impossible.

Similarly, if $p$ is strictly positive, then the minimum of $p$ can be only achieved in the region

$$
\left[\frac{\partial h}{\partial x} \geqslant 0\right]=\left\{(x, y) \in \Omega \mid \frac{\partial h}{\partial x}(x, y) \geqslant 0\right\}
$$

Remark 10: It is clear that (89) holds for instance when $\Omega$ is a smooth domain symmetric with respect to the $y$-axis and 


$$
h(-x, y)=h(x, y) \text { on } \partial \Omega
$$

In the following we show that the shape of the pressure $p$ can be more complicated than in the one dimensional case, by proving that $p$ can achieve its maximum (resp. minimum) at more than one point.

Let us begin with the following result:

Proposition 2: Assume that $(3)(4)(17)(18)(64)(65)(67)(68)$ hold and assume also that $h, \rho, \Omega$ are smooth. Then there exists a unique smooth positive solution $p$ of (81)(82). Moreover if $\Omega$ is symmetric with respect to the $x$-axis and if $h$ satisfies

$$
h(x,-y)=h(x, y) \text { in } \Omega
$$

then

$$
p(x,-y)=p(x, y) \text { in } \Omega \text {. }
$$

Proof: The existence and uniqueness of $p$ results from Theorems 1 and 9. From the weak formulation of (81) we have for all $\xi \in H_{0}^{1}(\Omega)$

$$
\int_{\Omega} h^{3}(x, y) \rho(p(x, y)) \nabla p(x, y) \cdot \nabla \xi(x,-y) d x d y=\alpha \int_{\Omega} h(x, y) \rho(p(x, y)) \frac{\partial}{\partial x} \xi(x,-y) d x d y .
$$

(Note that if $\xi(x, y) \in H_{0}^{1}(\Omega), \xi(x,-y) \in H_{0}^{1}(\Omega)$ thanks to the symmetry of $\Omega$ ).

Changing $y$ into - $y$ in the above integrals and using (91) we get

$$
\int_{\Omega} h^{3}(x, y) \rho(p(x,-y)) \nabla p(x,-y) \cdot \nabla \xi(x, y) d x d y=\alpha \int_{\Omega} h(x, y) \rho(p(x,-y)) \frac{\partial}{\partial x} \xi(x, y) d x d y
$$

and thus $p(x,-y)$ is a weak solution of (81)(82). By uniqueness of such a solution (92) follows. 
Let us consider a domain $\Omega$, containing 0 , and symmetric with respect to the $x$-axis, (see the figure below).

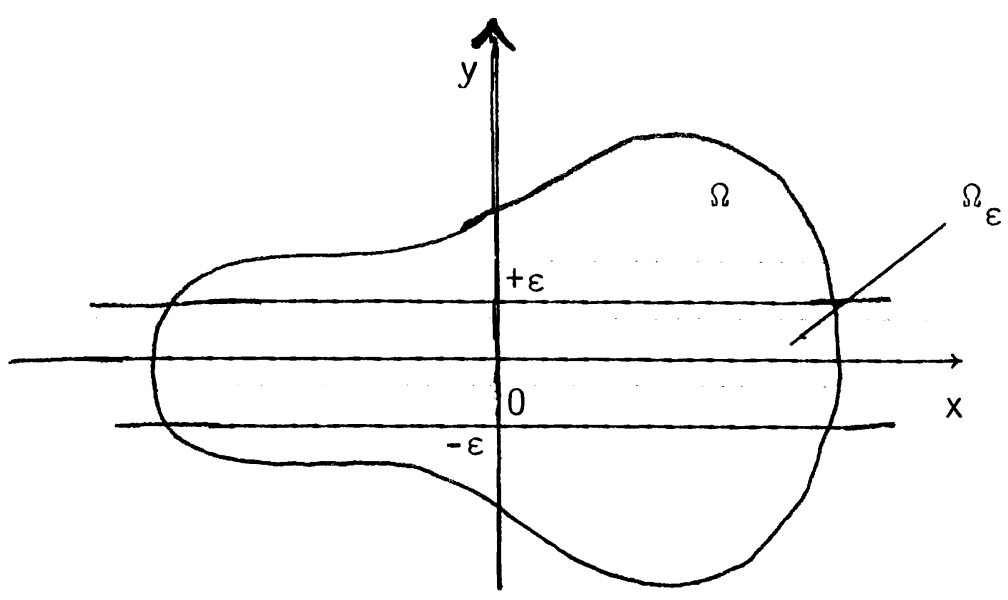

Let us denote by $\Omega_{\varepsilon}$ the strip

$$
\Omega_{\varepsilon}=\{(x, y) \in \Omega \mid y \in(-\varepsilon, \varepsilon)\}
$$

where we choose $\varepsilon$ small enough in such a way that $\Omega_{\varepsilon} \neq \Omega_{\text {. }}$.

Then we have the following:

Theorem 12: Assume that we are under the assumptions of the proposition 2. Moreover assume that $(84)(88)(89)(91)$ hold and that

$$
\frac{\partial h}{\partial x}(x, y) \equiv 0 \text { in } \Omega_{\varepsilon}
$$

Then the unique positive solution $p$ of (81)(82) achieves at least two maxima and at least two mimima outside of $\Omega_{\varepsilon}$ in $\Omega$ and no maximum nor minimum can be achieved in $\Omega_{\varepsilon} \cdot$

Proof: Thanks to theorem 12 (see remark 10) p achieves a maximum strictly greater than $p_{0}$ and a minimum strictly less than $p_{0}$ in $\Omega$. If the maximum say $M$ is achieved in $\Omega_{\varepsilon}$ then thanks to (81)(93) we have in $\Omega_{\varepsilon}$

$$
\nabla \cdot\left(h^{3} \rho(p) \nabla p\right)-\alpha h \rho^{\prime}(p) \frac{\partial p}{\partial x}=\alpha \frac{\partial h}{\partial x} \rho(p)=0
$$


and thus by the maximum principle $p \equiv M$ in $\Omega_{\varepsilon}$. But this contradicts

$$
p=p_{0}<M \quad \text { on } \quad \partial \Omega
$$

For the same reason $p$ cannot achieve its minimum in $\Omega_{\varepsilon}$. Thus $p$

achieves its maximum and its minimum outside of $\Omega_{\varepsilon}$. Now thanks to Proposition 2 we have (92) and it results that $p$ achieves its maximum and its minimum at at least two points symmetric with respect to the $x$-axis. This completes the proof of the theorem.

Acknowledgements: This work was done during the academic year 1984-1985 when I was visiting the Institute for Mathematics and its Applications. It is a pleasure for me to thank this Institution and their organizers for their support during the completion of this paper. I am also indebted to M. Luskin for several discussions which suggested some of the results presented here. 
[1] A. Cameron: Principles of lubrication - Longmans, London (1966).

[2] M. Chipot - J. Carrillo: On some nonlinear elliptic equations involving derivatives of the nonlinearity. Proceedings of the Royal Society of Edinburgh, 100A, (1985) p. 281-294.

[3] M. Chipot - M. Luskin: Existence and uniqueness of Solutions to Reynold's Lubrication Equation. To appear in Siam Journal of Analysis (1985).

[4] G. Cimatti: Nonlinear aspects of the theory of lubrication. Rend. di Mat. 3. (1980) p. 399-412.

[5] G. Cimatti: On certain nonlinear problems arising in the theory of Lubrication Appl. Math. Optim. 11 (1984) p. 227-245.

[6] D. Gilbarg, N.S. Trudinger: Elliptic Partial Differential Equations of Second order. Springer Verlag, 2nd. ed., 1985.

[7] A.G.M. Michel: Lubrication: Its principles and practice, Blackie, (1950).

[8] 0. Pinkus, B. Sternlicht: Theory of Hydrodynamics Lubrication, Mac Graw Hill, New York, (1961)

[9] M.H. Protter, H.F. Weinberger, Maximum principle in Differential Equations Springer-Verlag (1984).

[10] W. Rudin: Real and Complex Analysis, Mac Graw Hill. 


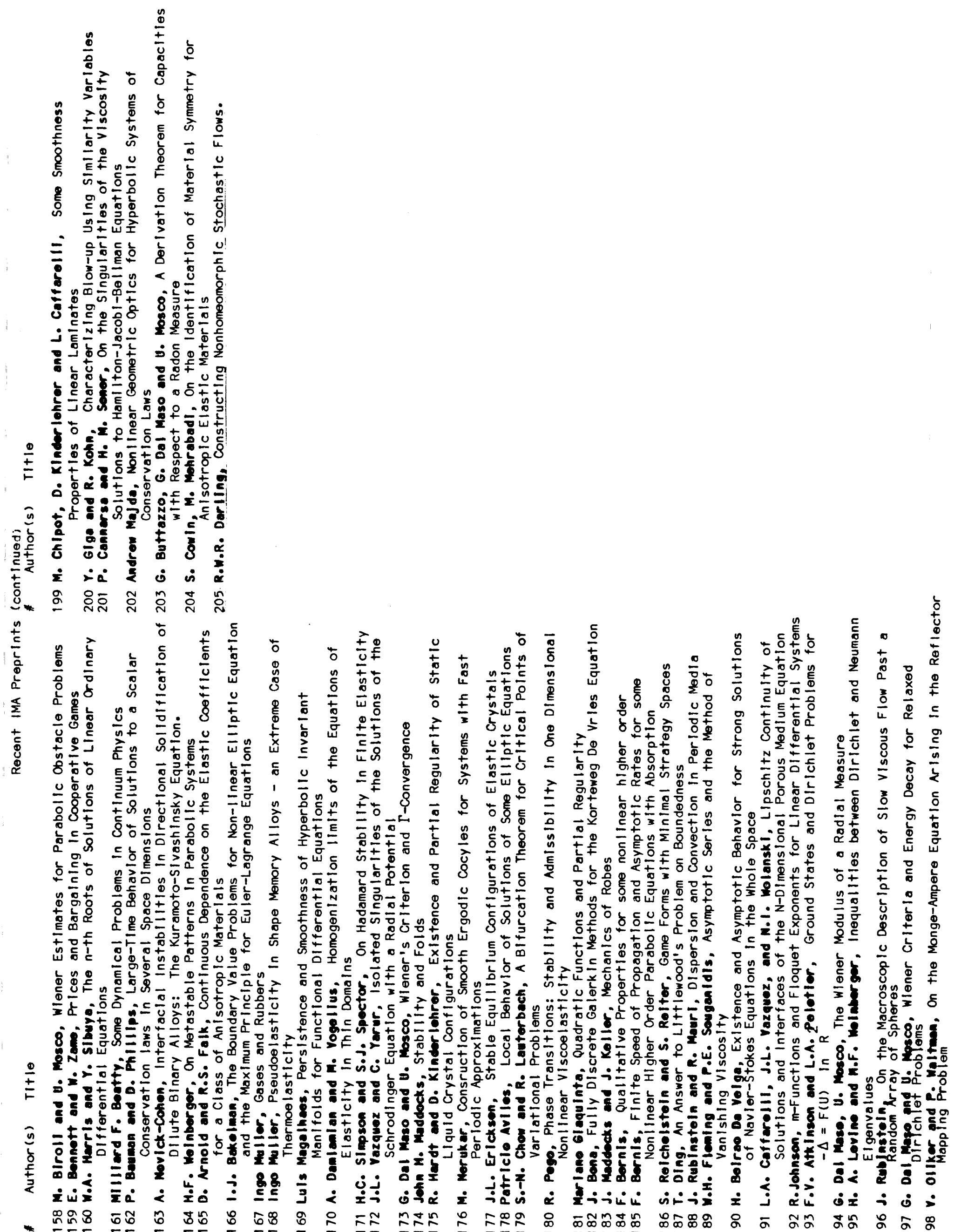


